\title{
Removal of False Negatives in Moving Object Detection Using RGB color space
}

\author{
Shailaja Surkutlawar \\ EXTC \\ Vivekananda education society of information technology \\ Mumbai, India
}

\begin{abstract}
Moving objects detection is a fundamental step in many vision based applications. Background subtraction is the typical method. Many background models have been introduced to deal with different problems. The method based on mixture of Gaussians is a good balance between accuracy and complexity, and is used frequently by many researchers. But it still cannot provide satisfied results in some cases. Video-surveillance and traffic analysis systems can be heavily improved using visionbased techniques to extract, manage and track objects in the scene. However, problems arise due to shadows. In particular, moving shadows can affect the correct localization, measurements and detection of moving objects. This work aims to present a technique for shadow detection and suppression used in a system using RGB color space for moving visual object detection and tracking. Experimental results show that the proposed approach can significantly enhance shadow suppression results and moving objects are detected.
\end{abstract}

Keywords-Moving objection detection; background subtraction; mixture of Gaussians MoG; Shadow detection; RGB color space.

\section{INTRODUCTION}

Moving Objects segmentation is a fundamental and critical task in many vision based applications, such as automated visual surveillance, human-machine interface, and very low-bandwidth telecommunications. A common approach is to perform background subtraction, which identifies moving objects from the difference between the current frame and a reference frame (which often called "background model"). The background model must be representation of the scene with no moving objects and must be kept regularly updated because for some cases, the background is changing with time.

To detect a moving object, a surveillance system usually utilizes background subtraction. The key of background subtraction is the background model. In the moving object detection process, one of the main challenges is to differentiate moving objects from their cast shadows. Moving cast shadows are usually misclassified as part of the moving object making the following stages, such as object classification or tracking, to perform inaccurate. The Gaussian mixture model (GMM) [1] represented the statistics of one pixel over time can cope

\author{
Prof. Ramesh Kulkarni \\ EXTC \\ Vivekananda education society of information technology \\ Mumbai, India
}

with multi-modal background distributions. However, a common problem for this approach is to find the right balance between the speed at which the model adapts to changing background, and the stability. The shadow points and the object points share two important visual features: motion model and detectability. Since the most common techniques for foreground object detection in dynamic scene are interframe difference or background suppression, all the moving points of both objects and shadows are detected at the same time.

Moreover, shadow points are usually adjacent to object points and with the more commonly used segmentation techniques shadows and objects are merged in a single blob. These aspects cause two important drawbacks called as false negatives: The former is that the object shape is falsified by shadows and all the measured geometrical properties are affected by an error (that varies during the day and when the luminance changes). This affects both the classification and the assessment of moving object position (normally given by the shape centroid), as, for instance, in traffic control systems that must evaluate the trajectories of vehicles and people on a road. The second problem is that the shadows of two or more objects can create a false adjacency between different objects. In this paper an approach of shadow detection and suppression based on RGB color space is defined.

The paper is organized as follows: Section II deals with the proposed method in which background subtraction using Gaussian Mixture Model to classify the pixels as background or foreground by thresholding the difference between the background image and the current image is explained, Section III deals with Post processing techniques for suppressing shadow using RGB color space, Section IV discusses the experimental results of shadow suppression techniques. Finally, the conclusion is given in Section V.

\section{PROPOSED METHOD}

In the proposed method shown in Fig. 1 the first step is to extract the foreground moving object from the background using mixture of Gaussian is used and then shadow suppression method using RGB color space is described on which area confidence is applied to extract the blobs to be detected. 


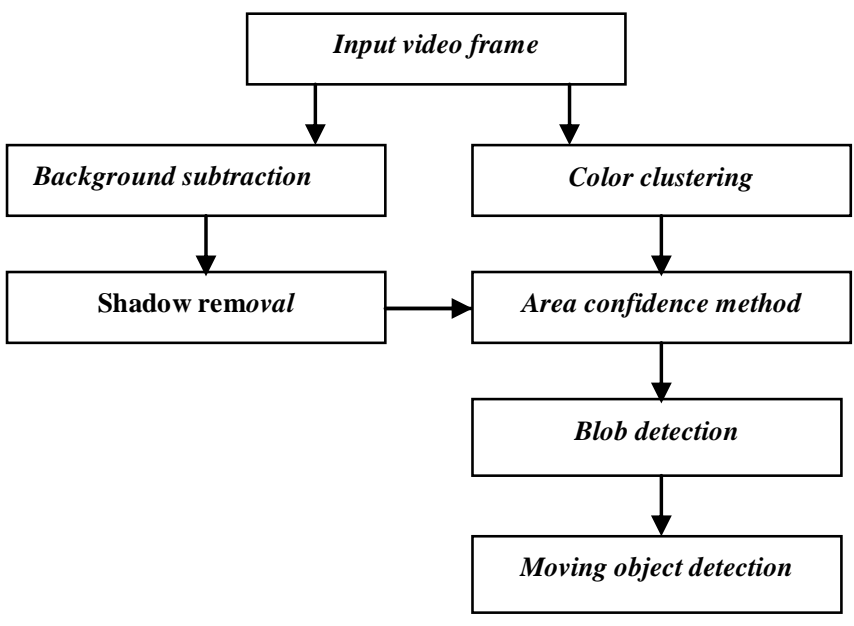

Fig.1. Block diagram of the proposed method

\section{A. Background subtraction $\backslash$}

In the model of Mixture of Gauss [1] [4] [5], the background is not a single frame without any moving objects. Gaussian Mixture Model (GMM) is thought to be one of the best background modeling methods and works well when gradual changes appear in the scene [2] [3]. The GMM method models the intensity of each pixel with a mixture of $\boldsymbol{k}$ Gaussian distributions. The probability that a certain pixel has a value $\boldsymbol{X}_{\boldsymbol{t}}$ at time can be written as

$\mathrm{P}\left(X_{t}\right)=\sum_{\mathrm{i}=1}^{\mathrm{k}} \omega_{i, t} \cdot \eta\left(X_{t}, \mu_{i, t}, \Sigma_{i, t}\right)$

Where $\boldsymbol{k}$ is the number of distributions (currently, 3 to 5 is used), $\boldsymbol{\omega}_{\boldsymbol{i}, \boldsymbol{t}}$ is the weight of the $\boldsymbol{k}^{\text {th }}$ Gaussian in the mixture at time $\boldsymbol{t}$ and $\boldsymbol{\eta}\left(\boldsymbol{X}_{\boldsymbol{t}}, \boldsymbol{\mu}_{i, t}, \boldsymbol{\Sigma}_{i, t}\right)$ the Gaussian probability density function.

$$
\eta\left(X_{t}, \mu_{i, t}, \Sigma_{i, t}\right)=\frac{1}{(2 \pi)^{3} /^{2}\left|\Sigma_{\mathrm{i}, t}\right|^{1} / 2} e^{\left\{\frac{-1\left(X_{t}-\mu_{i, t}\right)^{T} \sum_{i, t}^{-1}\left(X_{t}-\mu_{i, t}\right)}{2}\right\}}
$$

Where, $\boldsymbol{\mu}_{i, t}$ is the mean value and $\boldsymbol{\Sigma}_{i, t}$ is the covariance of the $\boldsymbol{k}^{\text {th }}$ Gaussian at time $\boldsymbol{t}$. For computational reasons, the covariance matrix is assumed to be of the form

$$
\sum_{k, t}=\sigma^{2} . I
$$

Where $\boldsymbol{\sigma}$ is the standard deviation.

This assumes that the red, green, and blue pixel values are independent and have the same variance, allowing us to avoid a costly matrix inversion at the expense of some accuracy.

Thus, the distribution of recently observed values of each pixel in the scene is characterized by a mixture of Gaussians. A new pixel value will, in general, be represented by one of the major components of the mixture model and used to update the model.

However, it fails when there are sharp changes, such as sudden illumination changes or sudden partial changes in the background. To tackle this problem, some improvement has been made in recent researches. In [6], every frame is processed on pixel level, region level and frame level with color and gradient information to overcome the problem caused by sudden illumination changes based on GMM. In [7], a hierarchical GMM using state models without temporal correlation on different scales is proposed to handle sharp changes. Zivkovic presented an improved GMM algorithm automatically fully adapting to the scene, by choosing the number of components for each pixel in an online procedure [8] [9], which leads to big improvement in reduced processing time and slight improvement in segmentation result.

If the pixel process could be considered a stationary process, a standard method for maximizing the likelihood of the observed data is expectation maximization. Unfortunately, each pixel process varies over time as the state of the world changes, therefore an approximate method which essentially treats each new observation as a sample set of size 1 and uses standard learning rules to integrate the new data.

If lighting changes occurred in a static scene, it would be necessary for the Gaussian to track those changes. If a static object was added to the scene and was not incorporated in to the background until it had been there longer than the previous object, the corresponding pixels could be considered foreground for arbitrarily long periods. This would lead to accumulated errors in the foreground estimation, resulting in poor tracking behavior. These factors suggest that more recent observations may be more important in determining the Gaussian parameter estimates. Since there is a mixture model for every pixel in the image, implementing an exact Expectation maximization algorithm on a window of recent data would be costly. Instead, we implement an on-line Kmeans approximation. Every new pixel value $\boldsymbol{X}_{\boldsymbol{t}}$, is checked against the existing $\boldsymbol{k}$ Gaussian distributions, until a match is found. A match is defined as a pixel value within 2.5 standard deviations of a distribution1. GMM algorithm can be summarized as:

- Initialize each pixel of the scene with $\boldsymbol{k}$ Gaussian distributions

- Every new pixel value $\boldsymbol{X}_{\boldsymbol{t}}$, is checked against the existing Gaussian distributions until a match is found.

- A match is defined as a pixel value within $\mathbf{2 . 5}$ standard deviations of a distribution.

- If none of $\boldsymbol{k}$-distributions match current pixel value, least probable distribution is go out.

- A new distribution with current value as mean value, an initially high variance, and low prior weight, is entered.

- Prior weights of $\boldsymbol{k}$ distributions at time adjusted as

$\omega_{k, t}=(1-\alpha) \omega_{k, t-1}+\alpha\left(M_{k, t}\right)$

- $\boldsymbol{\mu}_{\boldsymbol{t}}$ and $\boldsymbol{\sigma}$ for unmatched distributions remain the same.

- Parameters of distribution matching new observation are updated as : 


$$
\begin{aligned}
& \mu_{t}=(1-\rho) \mu_{t-1}+\rho\left(X_{t}\right) \\
& \sigma_{t}^{2}=(1-\rho) \sigma_{t-1}^{2}+\rho\left(X_{t}-\mu_{t}\right)^{T}\left(X_{t}-\mu_{t}\right) \\
& \rho=\alpha \eta\left(X_{t} \mid \mu_{t-1} \cdot \sigma_{t-1}\right)
\end{aligned}
$$

- Gaussians are ordered by the value of $\omega / \sigma$

- $1^{\text {st }}$ B distributions are chosen as background model, where

$$
B=\arg _{b}^{\min }\left(\sum_{k=1}^{b} \omega_{k}>T\right)
$$

\section{MOVING SHADOW SUPPRESSION USING RgB COLOR SPACE}

Shadows are due to the occlusion of light source by an object in the scene. In particular, that part of the object not illuminated is called self-shadow, while the area projected on the scene by the object is called cast shadow [10]. This last one is more properly called moving cast shadow if the object is moving.

\section{A. Normalized RGB color space}

The Normalized RGB space aims to separate the chromatic components from the brightness component. The red, green and blue channel can be transformed to their normalized counterpart by using the formulae

$l=R+G+B, r=R / l, \quad g=G / l, \quad b=B / l$

When $\boldsymbol{l} \neq \boldsymbol{0}$ and $\boldsymbol{r}=\boldsymbol{g}=\boldsymbol{b}=\boldsymbol{0}$ otherwise.

One of these normalized channels is redundant, since by definition $\boldsymbol{r}, \boldsymbol{g}$, and $\boldsymbol{b}$ sum up to 1 . Therefore, the Normalized RGB space is sufficiently represented by two chromatic components $\boldsymbol{r}$ and $\boldsymbol{g}$ and a brightness component 1 . Normalized RGB suffers from a problem inherent to the normalization namely noise sensor or compression noise at low intensities results in unstable chromatic components.

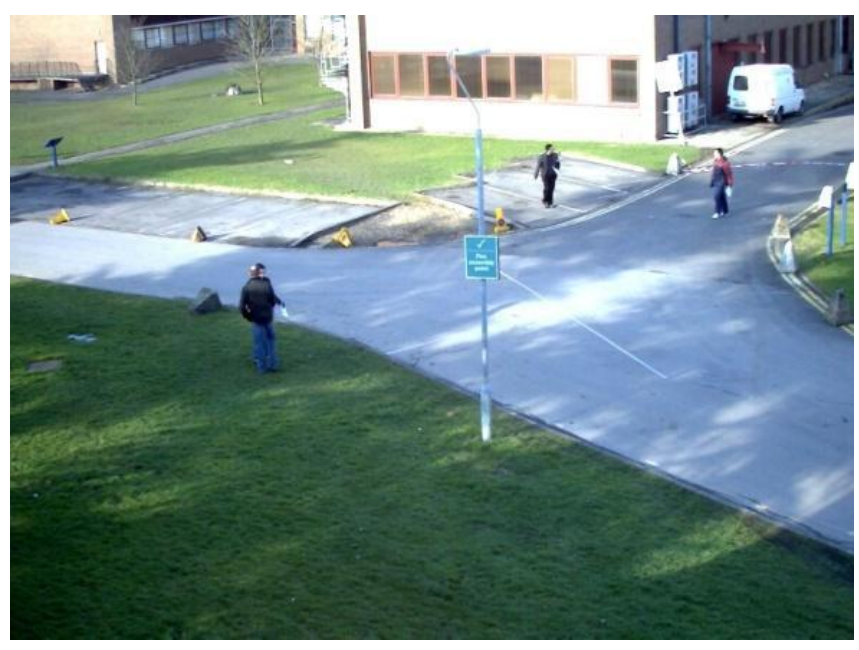

a) Original Frame 1
Under the consideration of saving computational cost, RGB space based method proposed by Horprasert in [4] is adopted. The basic idea in [4] is that shadow has similar chromaticity but lower brightness. For a given observed pixel value $\boldsymbol{I}_{i}$, a brightness distortion, $\alpha_{i}$, and a color distortion $\boldsymbol{C} \boldsymbol{D}_{i}$, is calculated by,

$\alpha_{i}=\arg _{\alpha_{i}} \min \left(I_{i}-\alpha_{i} E_{i}\right)^{2}$

$C D_{i}=\left\|I_{i}-\alpha_{i} E_{i}\right\|$

adopted. The basic idea in [4] is that shadow has similar chromaticity but lower brightness. For a given observed pixel

Where $\boldsymbol{E}$ is the expected chromaticity line, $\boldsymbol{\alpha}_{\boldsymbol{i}}$ equals 1 if the brightness of the given pixel in the current frame is the same as in the background image. $\boldsymbol{\alpha}_{\boldsymbol{i}}$, is less than 1 if it is darker and greater than 1 if it becomes brighter than the expected brightness. Then, the criteria for shadow pixels simply becomes,

$\left\{\begin{array}{c}\boldsymbol{\tau}_{\boldsymbol{a}}<\boldsymbol{\alpha}_{\boldsymbol{i}}<1 \\ \boldsymbol{C} \boldsymbol{D}_{\boldsymbol{i}}<\boldsymbol{\tau}_{\boldsymbol{C D}}\end{array}\right.$

In [11], $\boldsymbol{\tau}_{\boldsymbol{a}}$ and $\boldsymbol{\tau}_{C D}$ are predefined thresholds $\boldsymbol{\tau}_{\boldsymbol{a}}=\mathbf{0 . 7}$ and $\boldsymbol{\tau}_{C D}=5$, in our experiments.

\section{EXPERIMENTAL RESULTS}

Original frames for the experiments shown in Fig. a, Fig. b, Fig. c, Fig. d. On this original frames Background subtraction using GMM is applied, as a result background pixels, foreground pixels and some shadow pixels (falsely segmented as foreground pixels) shown as black and white respectively in Fig. e, Fig. f, Fig. g, Fig. h. Post processing techniques for shadow suppression using RGB color space applied on Fig. e, Fig. f, Fig. g, Fig. h and results shown in Fig. i, Fig. j, Fig. k, Fig. l. Results show that shadows and noise pixels eliminated in most of the images but suppression and blob size can be enhanced using HSV color space.

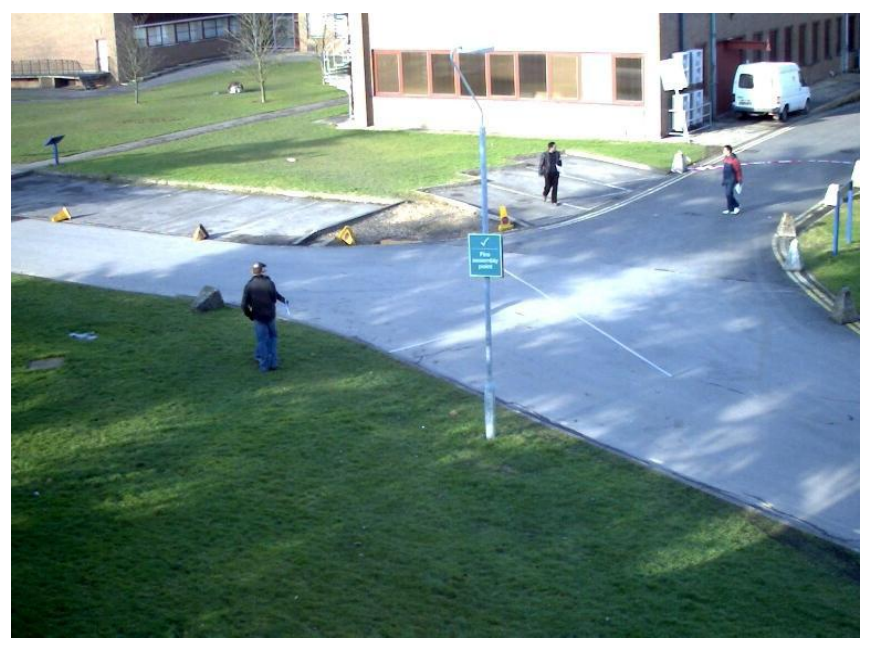

b) Original Frame 2 


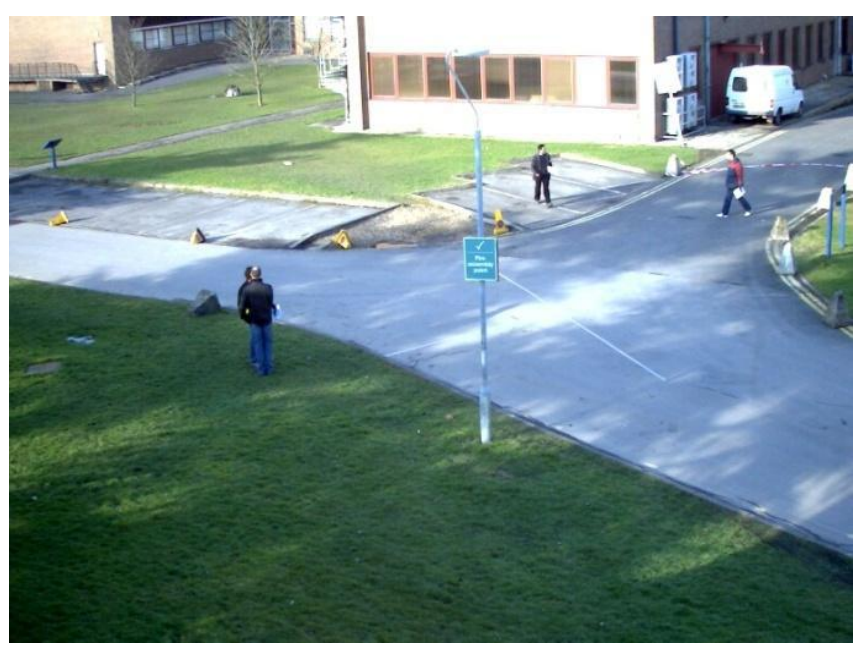

c) Original Frame 3

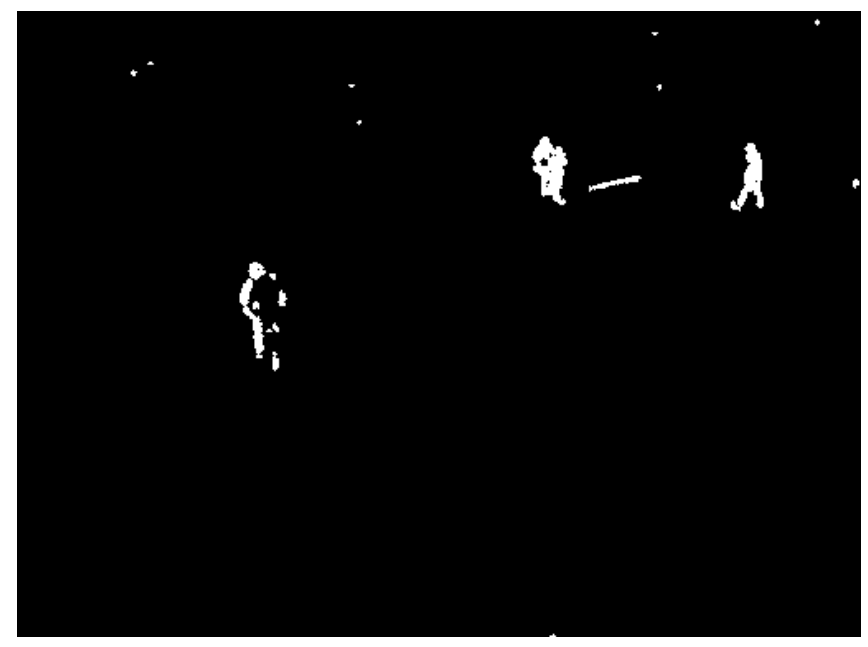

e) Background Subtraction of Frame 1

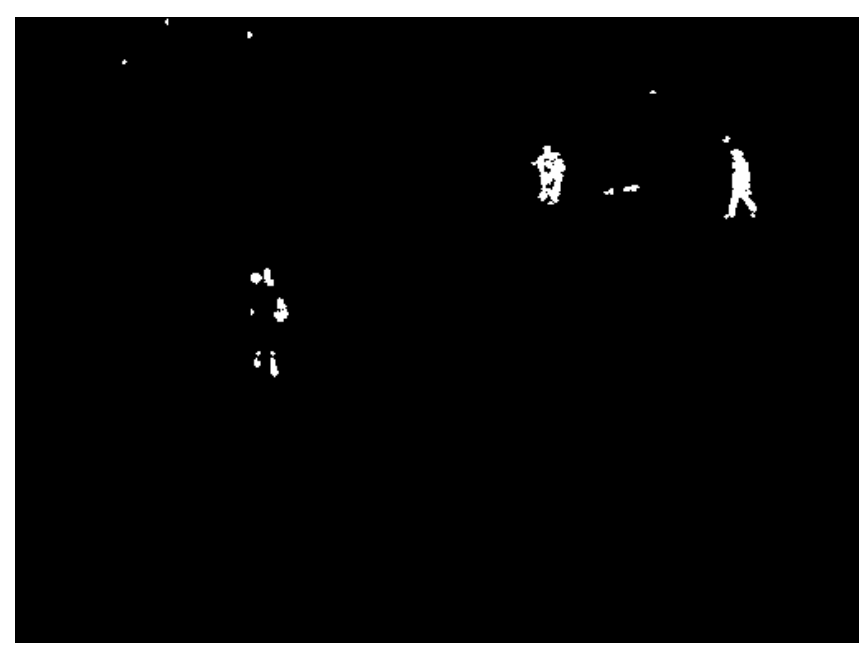

g) Background Subtraction of Frame 3

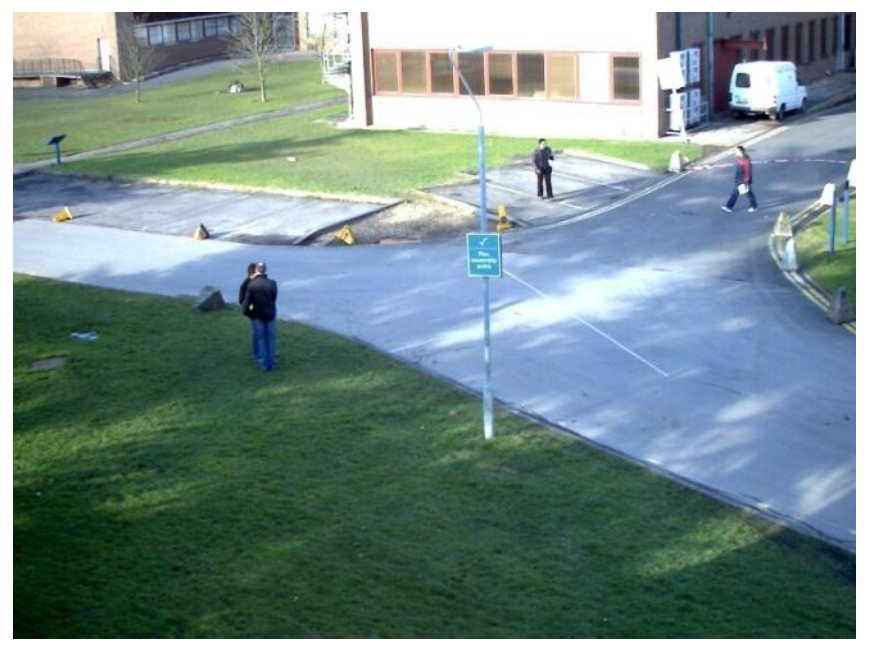

d) Original Frame 4

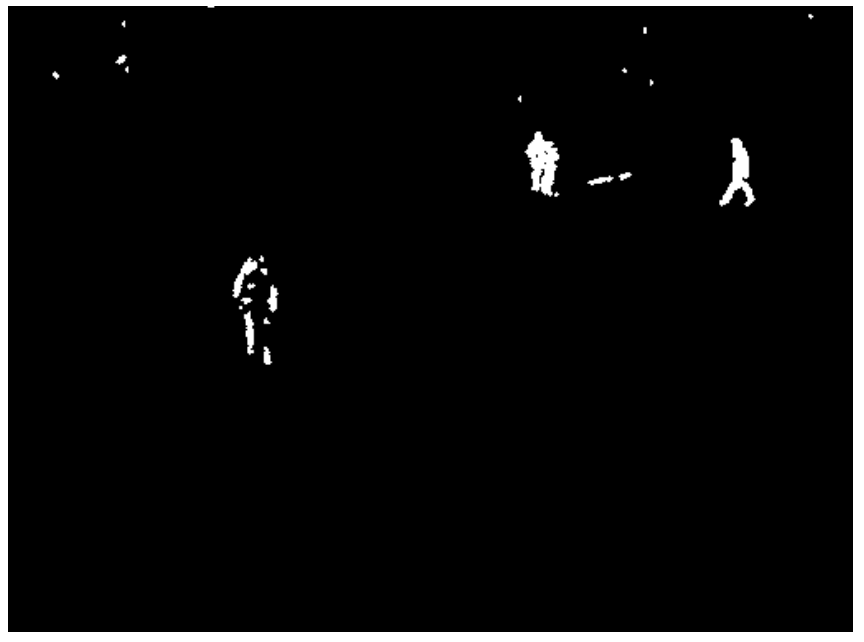

f) Background Subtraction of Frame 2

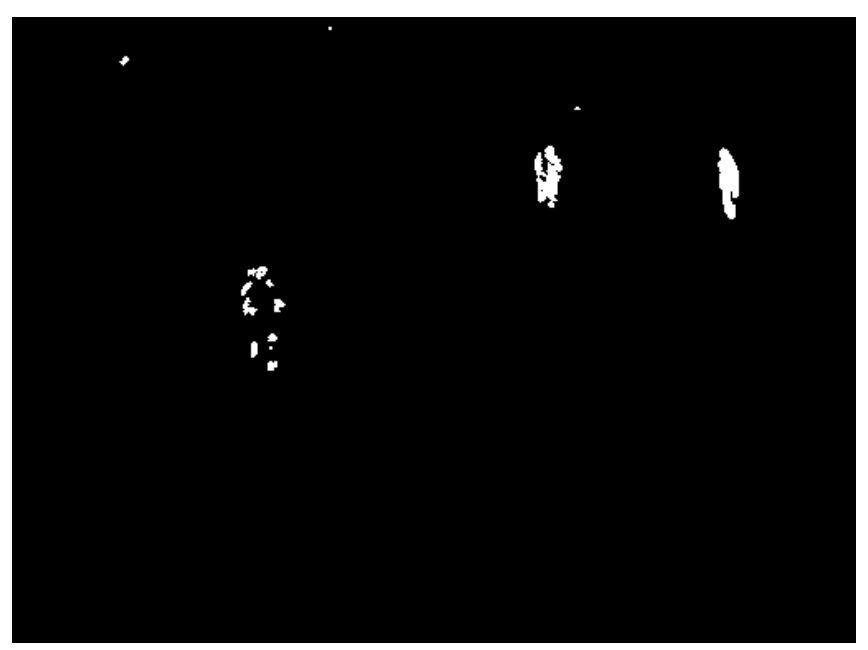

h) Background Subtraction of Frame 4 


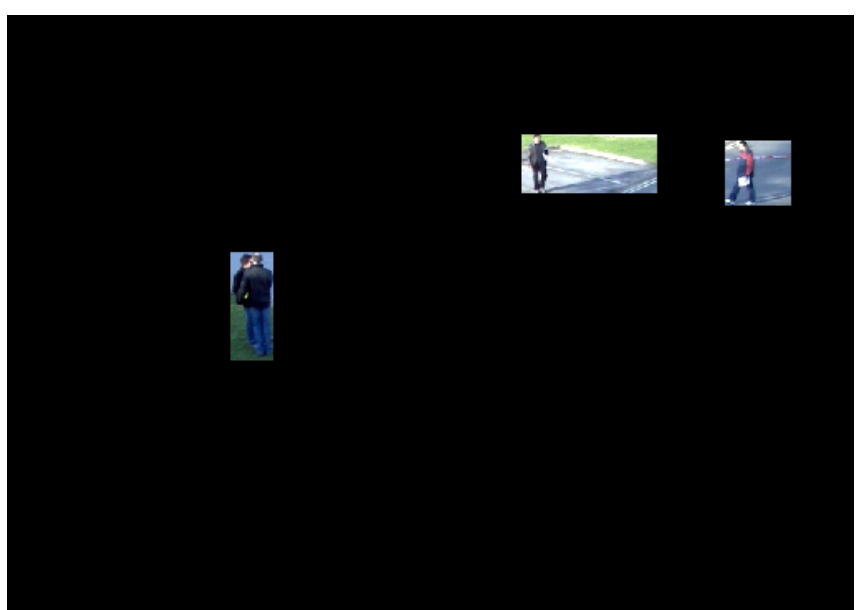

i) RGB Result Of Frame 1

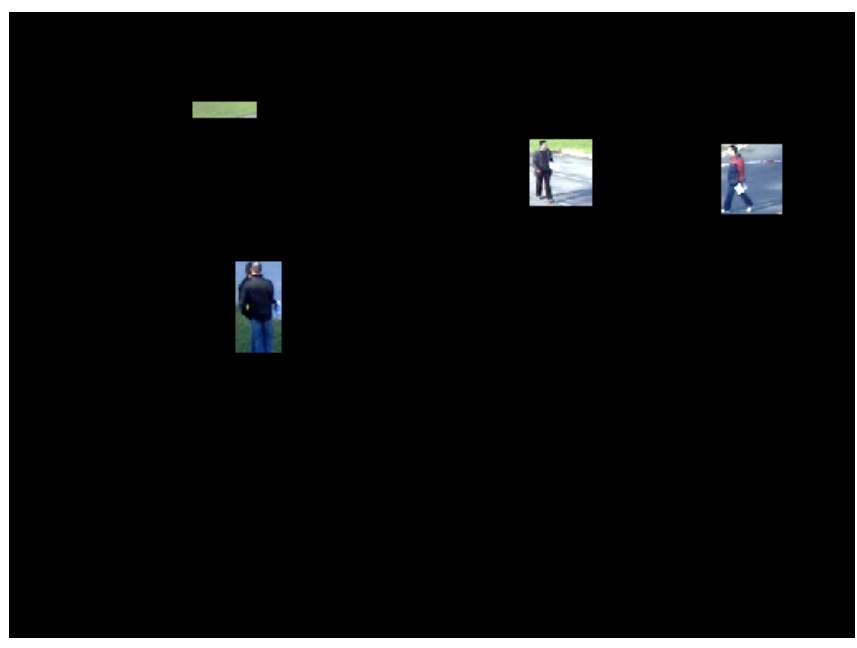

k) RGB Result Of Frame 3

\section{CONCLUSION}

Moving objects detection and segmentation is a fundamental step in many applications based on vision. Mixture of Gaussians is the frequently used method to subtracting moving objects from background. But its results are not good enough in some cases. In this paper, a postprocessing method is proposed to solve this problem. The results with more complete boundaries provided by the color clustering is used to verify the outputs of mixture of Gaussians, and thus two possible false segmentations can be corrected effectively. Moving shadow suppression using RGB and HSV colour spaces and small region median filter are also adopted. This paper compare shadow suppression results using RGB and HSV colour space and found that results of HSV are good over RGB colour space.

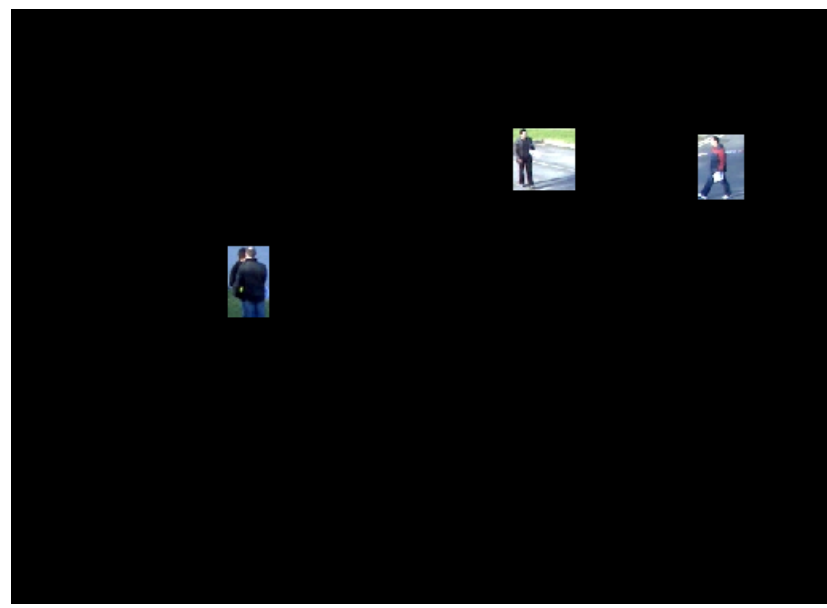

j) RGB Result Of Frame 2

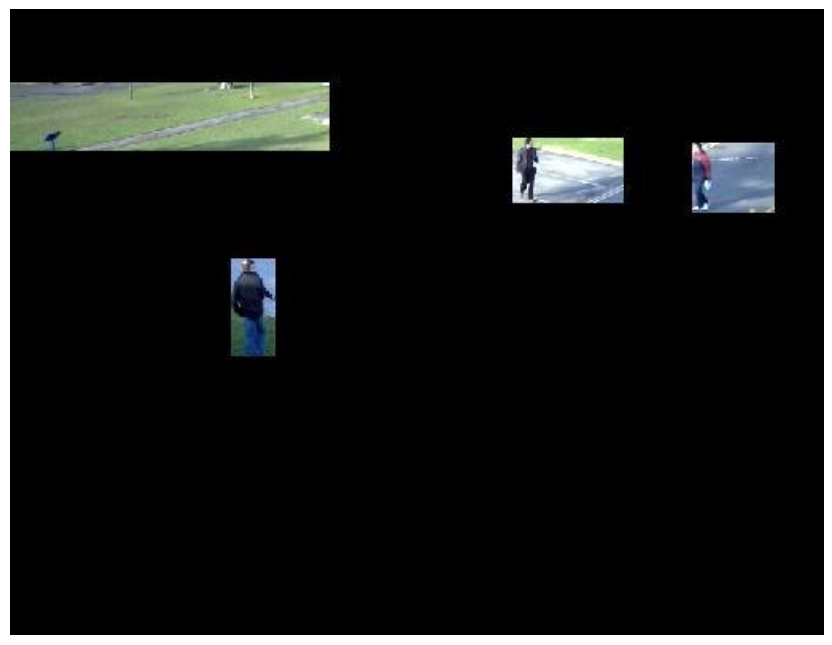

1) RGB Result Of Frame 4

\section{REFERENCES}

[1] C. Stauffer and W. E. L. Grimson, "Adaptive background mixture models for real-time tracking," in Proceedings of IEEE Conference on Computer Vision and Pattern Recognition. Ft. Collins, 1999: pp.246-252.

[2] C. Stauffer, and W. E. L Grimson, "Learning patterns of activity using real-time tracking," IEEE Trans. on PAMI, Vol. 22, No. 8,2000 pp. 747-757.

[3] P. Kumar, and A. Mittal, "Study of robust and intelligent surveillance in visible and multimodal framework," informatica, Vol. 31, 2007 pp. 447-461.

[4] Horprasert T, Harwood D, Davis L S. "A Statistical Approach for Real-time Robust Background Subtraction and Shadow Detection," in Proceedings of IEEE ICCV' 99 Frame-Rate Workshop, 1999, pp.1-19.

[5] KaewTraKulPong P., Bowden R, "An Improved Adaptive Background Mixture Model for Real time Tracking with Shadow Detection," in proceedings of 2nd European Workshop on Advanced Video Based Surveillance Systems, Sept 2001, Pages:1-5. 
[6] O.Javed, K.Shafique, and M .Shah., "A hierarchical approach to robust background subtraction using color and gradient information," In Workshop on Motion and Video Computing, Dec. 2002, pp. 22-27.

[7] Y.D.Sun, and B. Z. Yuan, "Hierarchical GMM to handle sharp changes in moving object detection," in Electronics Letters, Vol. 40, No. 13, 2004, pp. 801-802.

[8] Z.Zivkovic, "Improved adaptive gaussian mixture model for background subtraction," in proceedings ICPR, 2004.

[9] Z. Zivkovic and F.vander Heijden, " Efficient adaptive density estimation per image pixel for the task of background subtraction," Pattern Recognition Letters, Vol. 27,No. 7, 2006, pp. $773-780$.

[10] C. Jiang and M.O. Ward, "Shadow identification," in Proceedings of IEEE Int'l Conference on Computer Vision and Pattern Recognition, , 1992 ,pp. 606-612.

[11] I. Haritaoglu, D. Harwood, and L.S. Davis, "W4: Real-time surveillance of people and their activities,"in proceedings of IEEE Transactions on Pattern Analysis and Machine Intelligence, vol. 22, no. 8, Aug. 2000, pp. 809-830.

[12] J. Stauder, R. Mech, and J. Ostermann, "Detection of moving cast shadows for object segmentation,"in proceedings of IEEE Transactions on Multimedia, vol. 1, no. 1, Mar. 1999, pp. 65-76.
[13] M. Kilger, "A shadow handler in a video-based real-time traffic monitoring system," in Proceedings of IEEE Workshop on Applications of Computer Vision, 1992, pp. 11-18.

[14] A. Elgammal, D. Harwood, and L.S. Davis, "Non-parametric model for background subtraction," in Proceedings of IEEE ICCV'99 FRAME-RATE Workshop, 1999.

[15] T. Horprasert, D. Harwood, and L.S. Davis, "A Statistical approach for real-time robust background subtraction and shadow detection," in Proceedings of IEEE ICCV'99 FRAMERATE Workshop, 1999.

[16] R. Cucchiara, C. Grana, M. Piccardi, and A. Prati, "Statistical and knowledge-based moving object detection in traffic scene," in Proceedings of IEEE Int' 1 Conference on Intelligent Transportation Systems, Oct. 2000, pp. 27-32.

[17] I. Mikic, P. Cosman, G. Kogut, and M.M. Trivedi, "Moving shadow and object detection in traffic scenes," in Proceedings of Int'l Conference on Pattern Recognition, Sept. 2000.

[18] N. Herodotou, K.N. Plataniotis, and A.N. Venetsanopoulos, "A color segmentation scheme for object-based video coding," in Proceedings of the IEEE Symposium on Advances in Digital Filtering and Signal Processing, 1998, pp. 25-29. 